\title{
DEGRADAÇÃO E FORMAÇÃO DE RESÍDUOS NÃO-EXTRAÍVEIS OU LIGADOS DO HERBICIDA ATRAZINA EM SOLO'
}

\author{
LIA EMI NAKAGAWA² e MARA MERCEDES DE ANDRÉA ${ }^{3}$
}

\begin{abstract}
RESUMO - A persistência de um pesticida no solo depende de processos de dissipação, como a degradação, que pode estar relacionada com o metabolismo microbiano. Com o objetivo de se avaliar os mecanismos de dissipação do herbicida atrazina no solo e a importância dos microrganismos neste processo, avaliou-se sua mineralização, degradação intermediária e formação de resíduos não-extraíveis ou ligados ao solo após aplicação de ${ }^{14} \mathrm{C}$-atrazina em solo Glei Húmico. Os processos foram quantificados através de técnicas radiométricas e cromatográficas em solo natural, solo esterilizado e solo esterilizado e infectado com Pseudomonas putida. Observou-se a importância dos microrganismos através dos estudos de biomineralização nos quais detectou-se mineralização de ${ }^{14} \mathrm{C}$-atrazina somente em solo natural (cerca de 15\%). Entretanto, houve formação de metabólitos extraíveis de atrazina, tanto em solo esterilizado $(67 \%)$ como em solo natural (75\%), o que indica que este processo não dependeu apenas da presença de microrganismos. Já os resíduos não-extraíveis foram formados em maior quantidade (cerca de $56 \%$ ) no solo esterilizado.

Termos para indexação: ação dos pesticidas, metabólitos, microrganismos, mineralização.
\end{abstract}

\section{DEGRADATION AND FORMATION OF NON-EXTRACTABLE OR BOUND RESIDUES OF THE ATRAZINE HERBICIDE IN SOIL}

\begin{abstract}
The persistence of a pesticide in soil depends on the dissipation processes, as degradation, that can be related with microbial metabolism. Aiming to evaluate the mechanisms of atrazine dissipation in soil, and the importance of the microorganisms in this process, its mineralization, intermediate degradation and non-extractable or bound residues formation were evaluated after the application of ${ }^{14} \mathrm{C}$-atrazine in a Gley Humic soil. The processes were quantified by radiometric and chromatographic techniques in natural, sterilized and sterilized soil samples inoculated with Pseudomonas putida. The importance of microorganisms was observed through biomineralization studies, because the mineralization of ${ }^{14} \mathrm{C}$-atrazine was detected only in natural soil (about $15 \%$ ). However, formation of extractable metabolites of atrazine was detected both in sterilized $(67 \%)$ and natural $(75 \%)$ soil samples, indicating that these processes were not dependent only on the presence of microorganisms. On the other hand, bound residues were formed in higher amount (about 56\%) in sterilized soil.
\end{abstract}

Index terms: pesticide actions, metabolites, microorganisms, mineralization.

\section{INTRODUÇÃO}

A persistência de um pesticida no solo depende dos processos de dissipação, ou seja, dos proces-

\footnotetext{
${ }^{1}$ Aceito para publicação em 24 de março de 2000. Extraído da dissertação de mestrado do primeiro autor, apresentada ao IB/USP.

${ }^{2}$ Biól., M.Sc., Instituto Biológico, Centro de Proteção Ambiental, Caixa Postal 12.898, CEP 04010-970 São Paulo. E-mail: nakagawa@biologico.br

${ }^{3}$ Biól., Dra., Instituto Biológico, Centro de Proteção Ambiental. E-mail: andrea@biologico.br
}

sos de transferência, como: evaporação, lixiviação, escoamento superficial, absorção por plantas, e também, da taxa de degradação do pesticida (GrahamBryce, 1981). Os processos de degradação podem ser químicos, físicos ou biológicos, e podem resultar na mineralização total do pesticida ou na sua conversão em metabólitos. Entre esses processos, maior importância tem sido atribuída à degradação biológica, que está principalmente relacionada com os microrganismos presentes no solo (Graham-Bryce, 1981).

Durante o processo de dissipação, uma parte do pesticida aplicado inicialmente pode permanecer no 
solo na forma de resíduos. Esses resíduos, que podem ser o próprio composto ou seus metabólitos, podem estar na forma extraível ou ligada ao solo (resíduo não-extraível) (Calderbank, 1989). O resíduo ligado é formado por meio de ligações, como as pontes de hidrogênio, a troca catiônica, etc., entre a molécula do pesticida e a matéria orgânica do solo. A formação de resíduo ligado pode ter como conseqüências a diminuição da degradação do pesticida (Calderbank, 1989), a perda da sua atividade biológica (Kaufman, 1976) e da sua identidade química (Calderbank, 1989), a alteração da sua absorção por plantas, e a alteração do escoamento superficial e da lixiviação desses pesticidas.

A atrazina é um herbicida de pré-emergência que faz parte do grupo químico das triazinas, cuja principal via de degradação no solo, assim como das cloro-s-triazinas em geral, tem sido apontada como a hidrólise química a seus correspondentes hidroxi-striazinas (Kaufman, 1974). Entretanto, trabalhos mais recentes mostram que o mecanismo primário para dissipação de atrazina do ambiente se dá pela degradação biológica (Radosevich et al., 1995). Além da hidroxilação, que anula a fitotoxicidade, o metabolismo da atrazina em solo envolve, também, desalquilação e quebra do anel triazínico (Esser et al., 1975).

A formação de resíduos não-extraíveis ou ligados de atrazina foi observada por vários autores (Kruger et al., 1993; Assaf \& Turco, 1994; Blumhorst \&Weber, 1994; Nakagawa et al., 1995; Barriuso \& Koskinen, 1996; Kruger, et al., 1997; Andréa et al., 1997). No Brasil, Nakagawa et al. (1996) verificaram a formação de $61 \%$ e $82 \%$ de resíduos ligados do herbicida atrazina, 400 dias após a aplicação em solo Glei Húmico e Latossolo Vermelho-Escuro, respectivamente. Bertin et al. (1990) observaram que da atrazina aplicada, $28 \%$ se tornaram ligados ao solo.

A despeito da sua aparente degradabilidade, e devido ao seu uso extensivo, há relatos de contaminação de ecossistemas terrestres com atrazina, que pôde ser encontrada tanto em águas superficiais como de profundidade, em muitos países (Mougin et al., 1994).

Desta forma, estudos sobre dissipação de pesticidas são importantes por fornecerem indicações quanto à potencialidade de contaminação com resíduos e sobre sua persistência no ambiente agrícola.
Este trabalho avaliou a importância da microbiota de solo Glei Húmico nos processos de biomineralização, degradação intermediária e formação de resíduos que permanecem no solo mesmo após extração exaustiva.

\section{MATERIAL E MÉTODOS}

A degradação da atrazina foi estudada em triplicatas de solo Glei Húmico tratado com ${ }^{14} \mathrm{C}$-atrazina diluído em atrazina grau técnico e mantido em laboratório, durante 56 dias.

\section{Atrazina}

Solução-estoque de ${ }^{14} \mathrm{C}$-atrazina (uniformemente marcado no anel) com atividade específica de 270,1 $\mathrm{MBq} \mathrm{mmol}^{-1}$ e $97,8 \%$ de pureza radioquímica, obtida através de Agricur (São Paulo, SP), foi dissolvida em acetona, resultando em uma solução com atividade de $217,56 \mathrm{kBq}$ de ${ }^{14} \mathrm{C}$-atrazina $\mathrm{mL}^{-1}$. As soluções-estoque de $1 \mathrm{mg} \mathrm{mL}^{-1} \mathrm{de}$ atrazina grau técnico e seus metabólitos (hidroxiatrazina, desisopropilatrazina e desetilatrazina) foram preparadas em acetona. A solução de trabalho continha $5,66 \mathrm{kBq}$ de ${ }^{14} \mathrm{C}$-atrazina e $48 \mu \mathrm{g}$ do produto técnico por $\mathrm{mL}$ de acetona.

\section{Solo}

Utilizou-se solo Glei Húmico $(\mathrm{GH})$ proveniente do campo experimental do Instituto Biológico de São Paulo, com as seguintes características: $\mathrm{pH} 4,8 ; 36 \mathrm{~g} \mathrm{dm}^{-3}$ de matéria orgânica; $660 \mathrm{~g} \mathrm{~kg}^{-1}$ de argila; $60 \mathrm{~g} \mathrm{~kg}^{-1}$ de silte, e $280 \mathrm{~g} \mathrm{~kg}^{-1}$ de areia. Amostras de solo foram usadas nos estudos após esterilização por calor úmido (em autoclave a $120^{\circ} \mathrm{C}$ por 30 minutos, durante três dias consecutivos), esterilizadas e infectadas com Pseudomonas putida, ou solo natural.

A atividade microbiana nas amostras foi medida antes do início e ao final dos experimentos, por meio de radiorrespirometria, e os microrganismos foram quantificados pela técnica de contagem do número de unidades formadoras de colônias (UFC). Na radiorrespirometria, triplicatas equivalentes a $30 \mathrm{~g}$ de solo secado ao ar foram colocadas em frascos biométricos (Bartha \& Pramer, 1965), aplicou-se $1,0 \mathrm{~mL}$ de solução de ${ }^{14} \mathrm{C}$-glicose $(1,85 \mathrm{kBq}$ e $\left.0,9 \mathrm{mg} \mathrm{mL}^{-1}\right)$ e $10 \mathrm{~mL}^{\mathrm{de} \mathrm{KOH}}(0,2 \mathrm{M})$ foram colocados nos braços laterais dos frascos para coleta do ${ }^{14} \mathrm{CO}_{2}$ produzido a partir da ${ }^{14} \mathrm{C}$-glicose (Andréa \& Wiendl, 1995). Após 24 horas, a solução de álcali foi coletada, e alíquotas de $1 \mathrm{~mL}$ foram analisadas por espectrometria de cintilação líquida (LSC) em aparelho Packard LS 1600, após adição de solução cintiladora de Mesquita \& Rüegg (1984). 
A quantificação de microrganismos (UFC) foi efetuada através de suspensão de $10 \mathrm{~g}$ de solo em $100 \mathrm{~mL}$ de água destilada estéril (esterilização em autoclave a $120^{\circ} \mathrm{C}$ por 20 minutos), à qual foram adicionados $100 \mathrm{mg}$ de pirofosfato de sódio. Após 20 minutos de agitação, esta suspensão foi submetida a diluição seriada e plaqueamento em meio sólido de ágar nutritivo contendo $0,5 \mathrm{~g}$ de peptona, $0,3 \mathrm{~g}$ de extrato de carne, e 1,8 g de ágar/100 mL de água destilada estéril. Após 24 horas, realizou-se a contagem das colônias formadas.

\section{Dissipação de ${ }^{14} \mathrm{C}$-atrazina em solo}

A dissipação do herbicida foi estudada em laboratório, com três amostras de $50 \mathrm{~g}$ de solo de todos os tratamentos após aplicação de $0,192 \mathrm{kBq}$ e $1,56 \mu \mathrm{g}$ de solução de ${ }^{14} \mathrm{C}$-atrazina $\mathrm{g}^{-1}$, e colocadas em frascos de vidro com tampa rosqueável. Os frascos foram incubados em câmara de temperatura controlada (Fanen M347-6), a aproximadamente $29^{\circ} \mathrm{C}$, durante zero, 28 ou 56 dias.

A biomineralização da ${ }^{14} \mathrm{C}$-atrazina a ${ }^{14} \mathrm{CO}_{2}$ foi medida mediante captura de ${ }^{14} \mathrm{CO}_{2}$ em $10 \mathrm{~mL}$ de solução de $\mathrm{KOH}$ 0,2 M, colocada em pequenos frascos no interior de cada frasco com solo. A solução de $\mathrm{KOH}$ foi coletada semanalmente, substituída por uma solução recém-preparada, e analisada (três alíquotas de $1 \mathrm{~mL}$ ) por LSC para obtenção da taxa total de mineralização durante o período do experimento.

Para determinação das quantidades de radiocarbono remanescente, o próprio solo foi removido e analisado quanto ao resíduo total, por meio da combustão de três subamostras de $500 \mathrm{mg}$ de solo em "Biological Oxidizer" OX 600, e análise por LSC conforme Andréa et al. (1997). O solo natural foi analisado após zero, 28 e 56 dias, e o solo esterilizado, após 28 e 56 dias; o solo com Pseudomonas putida, somente após 56 dias.

Os resíduos extraíveis foram determinados mediante extração de ( 3 x $) 30 \mathrm{~g}$ de solo com $150 \mathrm{~mL}$ de mistura de metanol:água (8:2) por soxhlet durante oito horas (duas vezes consecutivas). Uma alíquota de $25 \mathrm{~mL}$ de extrato de cada amostra foi concentrada em rotaevaporador, retomada em solução cintiladora e analisada por LSC. Outra alíquota dos extratos $(80 \mathrm{~mL})$ foi concentrada em rotaevaporador e retomada em $1,5 \mathrm{~mL}$ de metanol, que foram aplicados em cromatoplaca de sílica gel $\mathrm{F}_{254}$ (Merck), juntamente com padrões de atrazina e metabólitos. A cromatoplaca foi desenvolvida primeiramente em éter etílico, para separação da atrazina e dos metabólitos desetilatrazina e desisopropilatrazina, e a seguir, em tolueno:acetona:ácido acético (7:1:2), por aproximadamente $7 \mathrm{~cm}$, para deslocamento da hidroxiatrazina do ponto de aplicação. As cromatoplacas foram então submetidas a varredura em analisador linear de radioatividade (Berthold II LB 2728); as áreas ativas detectadas tiveram a sílica removida por raspagem, e suas atividades foram quantificadas por LSC, conforme Nakagawa et al. (1996). Os Rfs resultantes foram: 0,70 para atrazina; 0,60 para desetilatrazina; 0,54 para desisopropilatrazina, e 0,07 para hidroxiatrazina.

Para determinação dos resíduos não-extraíveis ou ligados, três subamostras de $0,5 \mathrm{~g}$ de solo extraído foram também submetidas a combustão, conforme descrito anteriormente.

\section{Dissipação de ${ }^{14} \mathrm{C}$-atrazina em meio de cultura}

Estudou-se também a dissipação do herbicida em $10 \mathrm{~mL}$ de meio líquido de cultura $(0,5 \mathrm{~g}$ de peptona, $0,3 \mathrm{~g}$ de extrato de carne/100 $\mathrm{mL}$ água) contidos em tubos de ensaio esterilizados, aos quais misturou-se $0,170 \mathrm{kBq}$ de ${ }^{14} \mathrm{C}$-atrazina $\mathrm{mL}^{-1}$ de meio cultura, e $1 \mathrm{~mL}$ de suspensão aquosa de solo Glei Húmico ou de meio de cultura com P. putida. Em uma espiral de metal presa à rolha dos tubos, adaptou-se um béquer, no qual colocou-se $1 \mathrm{~mL}$ de $\mathrm{KOH} \mathrm{0,2} \mathrm{M} \mathrm{para} \mathrm{coleta} \mathrm{do}{ }^{14} \mathrm{CO}_{2}$ produzido. Os tubos foram incubados em câmara de temperatura controlada a, aproximadamente, $29^{\circ} \mathrm{C}$ durante 63 dias, e semanalmente a solução de $\mathrm{KOH}$ foi coletada e analisada por cintilação líquida.

Os resultados foram analisados estatisticamente pelo cálculo de média e desvio-padrão.

\section{RESULTADOS E DISCUSSÃO}

A atividade microbiana dos solos foi previamente comprovada por meio de mineralização de ${ }^{14} \mathrm{C}$-glicose, tendo resultado em $33,4 \%$ e $25,7 \%$ nas amostras com cultura pura de $P$. putida no início do experimento (T.0) e 56 dias após a incubação (T.56), respectivamente. Nas amostras de solo natural, detectou-se bioatividade de $8,7 \%$ e $11,7 \%$ (T.0 e T.56, respectivamente). Como o número de UFC foi zero no solo Glei Húmico esterilizado (tanto no início do experimento, como após 56 dias de incubação) e, respectivamente, $9,9 \times 10^{6}$ e $1,1 \times 10^{6}$ UFC no solo apenas com P. putida, e $8,8 \times 10^{7}$ e $4,4 \times 10^{6}$ UFC no solo natural, provou-se que o processo de esterilização foi eficiente. Além disso, como a população de $P$. putida foi também capaz de produzir significativa quantidade de ${ }^{14} \mathrm{CO}_{2}$ a partir de ${ }^{14} \mathrm{C}$-glicose, verificou-se que as condições experimentais se mantiveram durante $\mathrm{o}$ período do estudo. 


\section{Dissipação da ${ }^{14} \mathrm{C}$-atrazina em solo}

A produção de ${ }^{14} \mathrm{CO}_{2}$ a partir de ${ }^{14} \mathrm{C}$-atrazina foi de $0,2 \%$ no solo esterilizado, $0,15 \%$ no solo com P. putida, e $15,4 \%$ no solo natural após 56 dias de incubação das amostras de solo (Fig. 1 e Tabela 1). Observou-se que a biomineralização de atrazina foi importante somente no solo natural, pois nas amostras de solo esterilizado e com $P$. putida apenas, esta produção praticamente não ocorreu. Portanto, a quebra do anel triazínico do herbicida foi consequiência de ação microbiana, conforme também observado por Esser et al. (1975) e Kruger et al. (1997).

Entretanto, no solo infectado apenas com P. putida, a produção de ${ }^{14} \mathrm{CO}_{2}$ foi semelhante à da amostra esterilizada (Tabela 1 ). Isto prova que a adição desta única espécie não resultou em maior biomineralização da atrazina, que só foi detectada no solo com a microbiota natural total.

Em relação à recuperação do radiocarbono a partir da ${ }^{14} \mathrm{C}$-atrazina, verificou-se que após 28 dias de incubação os resíduos extraíveis representaram $52,2 \%$ do radiocarbono presente inicialmente no solo esterilizado, e $57,5 \%$ no solo natural, enquanto os resíduos ligados representaram, respectivamente, $42,8 \%$ e 32,2\% (Tabela 1). Após 56 dias de incubação, a quantidade de resíduos extraíveis decresceu, e a de resíduos ligados aumentou, pois detectou-se o nível de $40,1 \%$ de radiocarbono extraível no solo esterilizado, de $38,4 \%$ no solo com $P$. putida, e $43,9 \%$

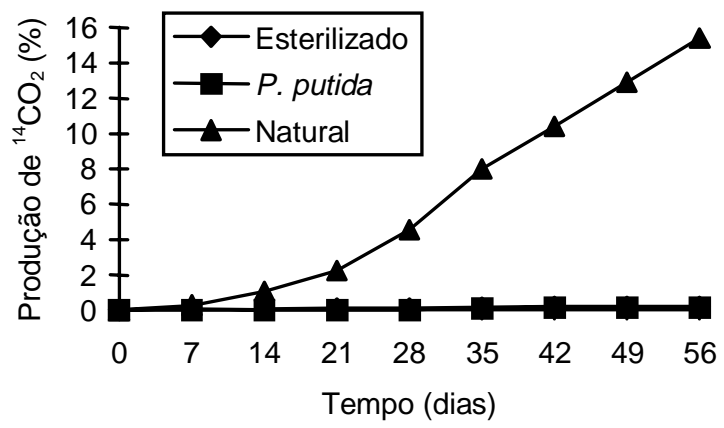

FIG. 1. ${ }^{14} \mathrm{CO}_{2}$ produzido em solo Glei Húmico esterilizado, com Pseudomonas putida e natural (porcentagem do radiocarbono aplicado no solo inicialmente: $0,19 \mathrm{kBq}$ e $1,56 \mu \mathrm{g}$ de atrazina/g de solo). no solo natural. Já as quantidades de resíduos ligados representaram $55,7 \%, 54,2 \%$ e $39,1 \%$, respectivamente (Tabela 1). Também Nakagawa et al. (1995) observaram anteriormente formação de $41,0 \%$ a $48,6 \%$ de resíduos ligados após 60 dias de incubação, conforme o tipo de solo, e Khan \& Behki (1990) citam formação de até $54 \%$ de resíduos ligados a partir de atrazina.

Desta forma, observou-se que a formação de resíduos ligados foi ligeiramente maior no solo esterilizado do que no solo natural (Tabela 1). Isto pode ter ocorrido em decorrência da própria mineralização, que pode ter diminuído a quantidade de resíduo disponível à formação de resíduos ligados. Além disso, este resultado indica que os microrganismos não foram diretamente responsáveis pela formação de resíduos ligados, fato já observado com outros pesticidas. Mostafa et al. (1982) demonstraram que a quantidade de resíduos ligados formados a partir de trifluralina foi maior no solo autoclavado (60\% a 88\%) do que no solo não-autoclavado (10\% a 33\%).

Quanto à degradação da atrazina a metabólitos, observou-se uma diminuição da atrazina e um aumento de seus metabólitos, principalmente da hidroxiatrazina. No início do experimento, detectaram-se $89,2 \%$ de atrazina e $3,1 \%$ de hidroxiatrazina, mas após 28 dias de incubação, a porcentagem de atrazina diminuiu para $44,5 \%$ e $40,7 \%$, e a de hidroxiatrazina aumentou para $25,9 \%$ e $35,4 \%$ nas amostras esterilizada e natural, respectivamente. Após 56 dias, essa tendência se acentuou, pois detectaram-se $32,5 \%, 47,1 \%$ e $24,9 \%$ de atrazina e $51,8 \%$, $39,5 \%$ e $51,4 \%$ de hidroxiatrazina nas amostras esterilizadas, com adição de $P$. putida, e natural, respectivamente (Tabela 2), indicando, assim, que o processo de degradação continuou com o tempo. Além da hidroxiatrazina, observou-se também a presença dos metabólitos desetilatrazina e desisopropilatrazina, embora em menor porcentagem, mas tanto aos 28 como aos 56 dias e em todas as amostras de solo. Ambos metabólitos parecem ter sido também degradados com o tempo e em todas as amostras, pois as porcentagens detectadas no T.56 diminuíram em relação ao T.28 (Tabela 2). Como a conversão da atrazina em metabólitos ocorreu tanto nas amostras de solo Glei Húmico esterilizado como no natural, provou-se que a presença de microrganismos não 
foi fator decisivo, e uma parte do processo de degradação pode ter sido puramente química, conforme também observado por outros autores (Blumhorst \& Weber, 1994; Andréa et al., 1997).

A degradação a hidroxiatrazina é importante do ponto de vista de rotação de culturas, porque representa destruição da fitotoxicidade da atrazina (Winkelmann \& Klaine, 1991; Blumhorst \& Weber, 1994). Além disso, a porcentagem de hidroxiatrazina pode também estar relacionada à formação de resíduos ligados, pois em estudo de Andréa et al. (1997), mais de $90 \%$ dos resíduos ligados de atrazina em solo eram hidroxiatrazina.

\section{Dissipação de ${ }^{14} \mathrm{C}$-atrazina em meio de cultura}

Após 63 dias de incubação de ${ }^{14} \mathrm{C}$-atrazina em meio de cultura contendo inóculo de suspensão de solo ou de $P$. putida, a produção de ${ }^{14} \mathrm{CO}_{2}$ foi muito baixa, variando de apenas $0,04 \%$ a $0,1 \%$ com inóculo de solo e inóculo de $P$. putida, respectivamente. Portanto, praticamente não houve mineralização da atrazina em meio de cultura, independentemente do

TABELA 1. Recuperação total do radiocarbono em solo Glei Húmico contendo resíduos ligados de ${ }^{14} \mathrm{C}$-atrazina; os dados representam porcentagens médias da atividade aplicada inicialmente \pm desvio-padrão ${ }^{1}$.

\begin{tabular}{clcccc}
\hline $\begin{array}{c}\text { Tempo de } \\
\text { incubação } \\
\text { (dias) }\end{array}$ & $\begin{array}{c}\text { Tratamento } \\
\text { do solo }\end{array}$ & $\begin{array}{c}{ }^{14} \mathrm{CO}_{2} \text { no } \\
\text { período }\end{array}$ & ${ }^{14} \mathrm{C}-$ extraível & ${ }^{14} \mathrm{C}$ - ligado & ${ }^{14} \mathrm{C}$ total \\
\hline \multirow{2}{*}{0} & Natural & 0 & $99,9 \pm 1,5$ & $3,2 \pm 0,1$ & $103,1 \pm 0,1$ \\
\multirow{2}{*}{28} & Esterilizado & $0,10 \pm 0,1$ & $52,2 \pm 9,1$ & $42,8 \pm 3,3$ & $95,2 \pm 11,1$ \\
& Natural & $5,00 \pm 0,4$ & $57,5 \pm 7,2$ & $32,2 \pm 0,5$ & $94,7 \pm 7,6$ \\
& & & & & \\
56 & Esterilizado & $0,20 \pm 0,1$ & $40,1 \pm 5,4$ & $55,7 \pm 2,6$ & $96,0 \pm 7,8$ \\
& P.putida & $0,15 \pm 0,03$ & $38,4 \pm 6,2$ & $54,2 \pm 2,3$ & $92,7 \pm 7,5$ \\
& Natural & $15,40 \pm 0,8$ & $43,9 \pm 0,6$ & $39,1 \pm 1,0$ & $98,4 \pm 2,0$ \\
\hline
\end{tabular}

${ }^{1} 0,19 \mathrm{kBq}$ e $1,56 \mu \mathrm{g}$ de atrazina/g de solo.

TABELA 2. Distribuição do radiocarbono presente nos extratos de solo Glei Húmico, por cromatografia em camada delgada; os dados representam médias da porcentagem em relação ao radiocarbono extraído \pm desvio-padrão.

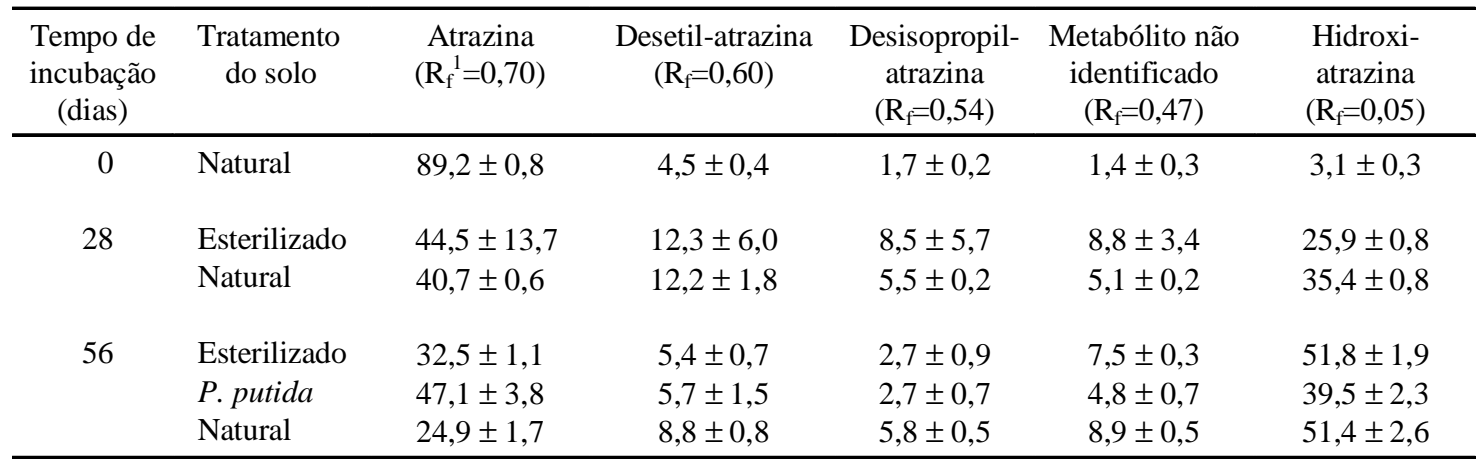

${ }^{1}$ Fator de retenção. 
inóculo, embora haja relatos de sua ocorrência (Mougin et al., 1994; Radosevich et al., 1995).

Comparando-se a mineralização de atrazina em solo e em meio de cultura, observou-se que ela ocorreu de forma significativa somente no solo natural, e não ocorreu no meio de cultura com inóculo de solo ou de $P$. putida. Portanto, a mineralização da atrazina ocorreu somente na presença de solo contendo a microbiota natural do solo.

\section{CONCLUSÕES}

1. Ocorre degradação da atrazina a hidroxiatrazina, desetilatrazina e desisopropilatrazina, paralelamente à mineralização e à formação de resíduos nãoextraíveis ou ligados, em solo Glei Húmico.

2. A mineralização do anel triazínico da molécula de atrazina ocorre somente em solo natural, não-esterilizado, e não ocorre em meio de cultura com microrganismos.

3. A degradação da atrazina em metabólitos intermediários e a formação de resíduos não-extraíveis não dependem da presença de microrganismos.

\section{AGRADECIMENTOS}

À CAPES, pela concessão de bolsa de estudos ao primeiro autor; à professora Dra. Vivian Pellizari, do Laboratório de Microbiologia Ambiental do ICB/USP, pela reativação das culturas de Pseudomonas putida.

\section{REFERÊNCIAS}

ANDRÉA, M.M.; MATALLO, M.B.; TOMITA, R.Y.; LUCHINI, L.C. Effect of the temperature on dissipation of $\left[{ }^{14} \mathrm{C}\right]$ atrazine in a Brazilian soil. Pesquisa Agropecuária Brasileira, Brasília, v.32, n.1, p.95-100, jan. 1997.

ANDRÉA, M.M.; WIENDL, F.M. Formation and biorelease of bound residues of pesticides in two Brazilian soils. I. $\left[{ }^{14} \mathrm{C}\right]$-lindane. Pesquisa Agropecuária Brasileira, Brasília, v.30, n.5, p.687694, maio 1995.

ASSAF, N.A.; TURCO, R.F. Influence of carbon and nitrogen application on the mineralization of the atrazine and its metabolites in soil. Pesticide Science, Chichester, v.41, p.41-47, 1994.

BARRIUSO, E.; KOSKINEN, W.C. Incorporating nonextracTable atrazine residues into soil size fractions as a function of time. Soil Science Society of America. Journal, Madison, v.60, p.150-157, 1996.

BARTHA, R.; PRAMER, D. Features of a flask and method for measuring the persistence and biological effects of pesticides in soil. Soil Science, Baltimore, v.100, n.1, p.68-70, 1965.

BERTIN, G.; SCHIAVON, M.; POTTIER, C. Plant bioavailability of "natural" and "model" humic acidbound $\left[{ }^{14} \mathrm{C}\right]$ atrazine residues. Toxicological and Environmental Chemistry, Chur, v.26, p.203-210, 1990.

BLUMHORST, M.R.; WEBER, J.B. Chemical versus microbial degradation of cyanazine and atrazine in soils. Pesticide Science, Chichester, v.42, p.79-84, 1994.

CALDERBANK, A. The occurrence and significance of bound pesticide residues in soil. Reviews of Environmental Contamination and Toxicology, New York, v.108, p.71-103, 1989.

ESSER, H.O.; DUPUIS, G.; EBERT. E.; MARCO, G.; VOGEL, C. S-triazines. In: KEARNEY, P.C.; KAUFMAN, D.D. (Ed.). Herbicides: chemistry, degradation, and mode of action. New York : M. Dekker, 1975. v.1, p.130-208.

GRAHAM-BRYCE, I.G. The behaviour of pesticides in soil. In: GREENLAND, D.J.; HAYES, M.H.B. (Ed.). The chemistry of soil processes. New York : J. Wiley, 1981. p.621-670.

KAUFMAN, D.D. Bound and conjugated pesticide residues. In: KAUFMAN, D.D.; STILL, G.G.; PAULSON, G.D.; BANDAL, S.K. (Ed.). Bound and conjugated pesticide residues. Washington : American Chemical Society, 1976. p.1-10. (ACS Symposium Series, 29).

KAUFMAN, D.D. Degradation of pesticides by soil microorganisms. In: GUENZI, W.D. (Ed.). Pesticides in soil and water. Madison : Soil Science Society of America, 1974. p.133-202.

KHAN, S.U.; BEHKI, R.M. Effects of Pseudomonas species on the release of bound ${ }^{14} \mathrm{C}$ residues from soil treated with $\left[{ }^{14} \mathrm{C}\right]$ atrazine. Journal of 
Agricultural and Food Chemistry, Washington, v.38, p.2090-2093, 1990.

KRUGER, E.L.; RICE, P.J.; ANHALT, J.C.; ANDERSON, T.A.; COATS, J.R. Organic chemicals in the environment: comparative fates of atrazine and deethylatrazine in sterile and non-sterile soils. Journal of Environmental Quality, Madison, v.26, p.95-101, 1997.

KRUGER, E.L.; SOMASUNDARAM, L.; KANWAR, R.S.; COATS, J.R. Persistence and degradation of $\left[{ }^{14} \mathrm{C}\right]$ atrazine and deisopropilatrazine as affected by soil depth and moisture conditions. Environmental Toxicology and Chemistry, Pensacola, v.12, p.19591967, 1993.

MESQUITA, T.B.; RÜEGG, E.F. Influência de agentes tensoativos na detecção da radiação beta. Ciência e Cultura, São Paulo, v.36, p.446-450, 1984.

MOSTAFA, I.Y.; ZAYED, S.M.A.D.; ADAM, Y.M.; ATTABY, H.S.H. Investigations on trifluralin binding to soil and possible uptake of bound residues by plants. Journal of Environmental Science and Health, New York, v.B17, n.3, p.265-275, 1982.

MOUGIN, C.; LAUGERO, C.; ASHTER, M.; DUBROCA, J.; FRASSE, P.; ASHTER, M. Biotransformation of the herbicide atrazine by the white rot fungus Phanerochaete chrysosporium.
Applied and Environmental Microbiology, Washington, v.60, n.2, p.705-708, 1994.

NAKAGAWA, L.E.; LUCHINI, L.C.; MUSUMECI, M.R.; ANDREA, M.M. Comportamento da atrazina em solos brasileiros em condições de laboratório. Pesquisa Agropecuária Brasileira, Brasília, v.30, n.4, p.471-476, abr. 1995.

NAKAGAWA, L.E.; LUCHINI, L.C.; MUSUMECI, M.R.; MATALLO, M.B. Behavior of atrazine in soils of tropical zone: degradation, mobility and uptake of atrazine residues from soils in a crop rotation system (maize/beans). Journal of Environmental Science and Health, New York, v.B31, n.2, p.203-224, 1996.

RADOSEVICH, M.; TRAINA, S.J.; HAO, Y.L.; TUOVINEN, O.H. Degradation and mineralization of atrazine by a soil bacterial isolate. Applied and Environmental Microbiology, Washington, v.61, n.1, p.297-302, 1995.

WINKELMANN, D.A.; KLAINE, S.J. Atrazine metabolite behaviour in soil-core microcosms: formation, disappearance, and bound residues. In: SOMASUNDARAM, L.; COATS, J.R. (Ed.). Pesticide transformation products fate and significance in the environment. Washington : American Chemical Society, 1991. p.75-92. (ACS Symposium Series 459). 